\title{
NOTE ON WEIGHTED CARLEMAN-TYPE INEQUALITY
}

CHAO-PING CHEN, WING-SUM CHEUNG, AND FENG QI

Received 30 October 2003 and in revised form 19 August 2004

A double inequality involving the constant $e$ is proved by using an inequality between the logarithmic mean and arithmetic mean. As an application, we generalize the weighted Carleman-type inequality.

\section{Introduction}

Let $p>1$ and $a_{n} \geq 0$ with $0<\sum_{n=1}^{\infty} a_{n}^{p}<\infty$. Then

$$
\sum_{n=1}^{\infty}\left(\frac{a_{1}+a_{2}+\cdots+a_{n}}{n}\right)^{p}<\left(\frac{p}{p-1}\right)^{p} \sum_{n=1}^{\infty} a_{n}^{p} .
$$

The constant $(p /(p-1))^{p}$ is the best possible.

Inequality (1.1) is due to Hardy [6, page 239].

Replacing $a_{n}$ in (1.1) by $a_{n}^{1 / p}$ for $n \in \mathbb{N}$, we obtain

$$
\sum_{n=1}^{\infty}\left(\frac{a_{1}^{1 / p}+a_{2}^{1 / p}+\cdots+a_{n}^{1 / p}}{n}\right)^{p}<\left(\frac{p}{p-1}\right)^{p} \sum_{n=1}^{\infty} a_{n} .
$$

In (1.2), letting $p \rightarrow \infty$, then the following Carleman inequality [6, page 249] is deduced:

$$
\sum_{n=1}^{\infty}\left(a_{1} a_{2} \cdots a_{n}\right)^{1 / n}<e \sum_{n=1}^{\infty} a_{n}
$$

where $a_{n} \geq 0$ for $n \in \mathbb{N}$ and $0<\sum_{n=1}^{\infty} a_{n}<\infty$. The constant $e$ is the best possible.

Carleman's inequality (1.3) was generalized in [6, page 256] by Hardy as follows. Let $a_{n} \geq 0, \lambda_{n}>0, \Lambda_{n}=\sum_{m=1}^{n} \lambda_{m}$ for $n \in \mathbb{N}$, and $0<\sum_{n=1}^{\infty} \lambda_{n} a_{n}<\infty$, then

$$
\sum_{n=1}^{\infty} \lambda_{n}\left(a_{1}^{\lambda_{1}} a_{2}^{\lambda_{2}} \cdots a_{n}^{\lambda_{n}}\right)^{1 / \Lambda_{n}}<e \sum_{n=1}^{\infty} \lambda_{n} a_{n} .
$$


Note that inequality (1.4) is usually referred to as a Carleman-type inequality or weighted Carleman-type inequality. In his original paper [5], Hardy himself said that it was Pölya who pointed out this inequality to him.

In several recent papers $[2,4,11,12,13,14,15]$, some strengthened and generalized results of (1.3) and (1.4) have been given by estimating the weight coefficient $(1+1 / n)^{n}$.

For information about the history of both Hardy's inequality and Carleman-type inequalities, please refer to $[7,9]$.

In this note, we will give a generalization of (1.4) as follows.

Theorem 1.1. Let $0<\lambda_{n+1} \leq \lambda_{n}$ with $\Lambda_{n}=\sum_{m=1}^{n} \lambda_{m} \geq 1$ and $\lim _{n \rightarrow \infty} \Lambda_{n}=\infty$, and let $a_{n} \geq$ 0 for $n \in \mathbb{N}$ satisfying $0<\sum_{n=1}^{\infty} \lambda_{n} a_{n}<\infty$. Then for $0<p \leq 1$,

$$
\begin{aligned}
& \sum_{n=1}^{\infty} \lambda_{n+1}\left(a_{1}^{\lambda_{1}} a_{2}^{\lambda_{2}} \cdots a_{n}^{\lambda_{n}}\right)^{1 / \Lambda_{n}} \\
& \quad \leq \frac{1}{p} \sum_{n=1}^{\infty}\left[\left(1+\frac{1}{\Lambda_{n} / \lambda_{n}}\right)^{p \Lambda_{n} / \lambda_{n}} \lambda_{n} a_{n}^{p} \Lambda_{n}^{p-1}\left(\sum_{k=1}^{n} \lambda_{k}\left(c_{k} a_{k}\right)^{p}\right)^{(1-p) / p}\right],
\end{aligned}
$$

in particular,

$$
\begin{aligned}
& \sum_{n=1}^{\infty} \lambda_{n+1}\left(a_{1}^{\lambda_{1}} a_{2}^{\lambda_{2}} \cdots a_{n}^{\lambda_{n}}\right)^{1 / \Lambda_{n}} \\
& \quad<\frac{e^{p}}{p} \sum_{n=1}^{\infty}\left[\left(1-\frac{1-2 / e}{\Lambda_{n} / \lambda_{n}}\right)^{p} \lambda_{n} a_{n}^{p} \Lambda_{n}^{p-1}\left(\sum_{k=1}^{n} \lambda_{k}\left(c_{k} a_{k}\right)^{p}\right)^{(1-p) / p}\right]
\end{aligned}
$$

where

$$
c_{k}^{\lambda_{k}}=\frac{\left(\Lambda_{k+1}\right)^{\Lambda_{k}}}{\left(\Lambda_{k}\right)^{\Lambda_{k-1}}} .
$$

Remark 1.2. In particular, taking in (1.6) $p=1$, we obtain the following strengthened Hardy's inequality:

$$
\sum_{n=1}^{\infty} \lambda_{n+1}\left(a_{1}^{\lambda_{1}} a_{2}^{\lambda_{2}} \cdots a_{n}^{\lambda_{n}}\right)^{1 / \Lambda_{n}}<e \sum_{n=1}^{\infty}\left(1-\frac{1-2 / e}{\Lambda_{n} / \lambda_{n}}\right) \lambda_{n} a_{n} .
$$

Taking in (1.8) $\lambda_{n} \equiv 1$, we obtain the following strengthened Carleman's inequality:

$$
\sum_{n=1}^{\infty}\left(a_{1} a_{2} \cdots a_{n}\right)^{1 / n}<e \sum_{n=1}^{\infty}\left(1-\frac{1-2 / e}{n}\right) a_{n} .
$$

\section{Lemma}

The well-known arithmetic mean $A(a, b)$ and logarithmic mean $L(a, b)$ of two positive numbers $a$ and $b$ are defined, respectively, for $a=b$ by $A(a, b)=L(a, b)=a$ and for $a \neq b$ 
by

$$
A(a, b)=\frac{a+b}{2}, \quad L(a, b)=\frac{b-a}{\ln b-\ln a} .
$$

For $a \neq b$, we have

$$
L(a, b)<A(a, b) .
$$

See [1] and the references therein.

Lemma 2.1. Let $x \geq 1$ be a real number. Then

$$
e\left(1-\frac{1 / 2}{x}\right)<\left(1+\frac{1}{x}\right)^{x} \leq e\left(1-\frac{1-2 / e}{x}\right) .
$$

The constants $1 / 2$ and $1-2 /$ e are best possible.

Proof. Inequality (2.3) is equivalent to

$$
1-\frac{2}{e} \leq x\left[1-\frac{1}{e}\left(1+\frac{1}{x}\right)^{x}\right]<\frac{1}{2} .
$$

Define a function $f$ for $x>0$ by

$$
f(x)=x\left[1-\frac{1}{e}\left(1+\frac{1}{x}\right)^{x}\right] .
$$

In order to prove (2.4), it is sufficient to show that the function $f$ is strictly increasing on $[1, \infty)$ and with

$$
f(1)=1-\frac{2}{e}, \quad \lim _{x \rightarrow \infty} f(x)=\frac{1}{2}
$$

The following proof shows that in fact $f^{\prime}(x)>0$ holds on $(0, \infty)$.

Easy computation yields

$$
e f^{\prime}(x)=e-[1+x g(x)]\left(1+\frac{1}{x}\right)^{x}
$$

where

$$
g(x)=\ln \left(1+\frac{1}{x}\right)-\frac{1}{x+1}=\frac{1}{L(x, x+1)}-\frac{1}{x+1} .
$$

Now we are in a position to prove $f^{\prime}(x)>0$, which is equivalent to

$$
h(x)=[1+x g(x)]\left(1+\frac{1}{x}\right)^{x}<e .
$$


Differentiation yields

$$
h^{\prime}(x)=\left[x g^{2}(x)+2 g(x)-\frac{1}{(x+1)^{2}}\right]\left(1+\frac{1}{x}\right)^{x} .
$$

In the following we show $h^{\prime}(x)>0$. Clearly, the equation

$$
x t^{2}+2 t-\frac{1}{(x+1)^{2}}=0
$$

has two roots

$$
t_{1,2}=\frac{-(x+1) \pm \sqrt{(x+1)^{2}+x}}{x(x+1)} .
$$

To prove $h^{\prime}(x)>0$, it is sufficient to show that

$$
\frac{-(x+1)+\sqrt{(x+1)^{2}+x}}{x(x+1)}=t_{2}<g(x)=\frac{1}{L(x, x+1)}-\frac{1}{x+1},
$$

which is equivalent to

$$
\frac{\sqrt{(x+1)^{2}+x}-1}{x(x+1)}<\frac{1}{L(x, x+1)} .
$$

Inequality (2.14) holds based on the following fact:

$$
\frac{\sqrt{(x+1)^{2}+x}-1}{x(x+1)}<\frac{2}{2 x+1}=\frac{1}{A(x, x+1)}<\frac{1}{L(x, x+1)} .
$$

Hence, the function $h$ is increasing on $(0, \infty)$, and then $h(x)<\lim _{x \rightarrow \infty} h(x)=e$. This means $f^{\prime}(x)>0$, and then

$$
1-\frac{2}{e}=f(1)<\lim _{x \rightarrow \infty} f(x)
$$

Using Maclaurin formula

$$
(1+t)^{1 / t}=e-\frac{e}{2} t+o(t)
$$

we have

$$
\lim _{n \rightarrow \infty} f(n)=\lim _{x \rightarrow \infty} f(x)=\lim _{t \rightarrow 0+} f\left(\frac{1}{t}\right)=\lim _{t \rightarrow 0+} \frac{(e t) / 2+o(t)}{\text { et }}=\frac{1}{2} .
$$

The proof of Lemma 2.1 is complete.

Remark 2.2. There are other very sharp estimates of the crucial factor $(1+1 / n)^{n}$ in [8] and the references therein. 


\section{Proof of Theorem 1.1}

By the power mean inequality, we have

$$
\prod_{m=1}^{n} \alpha_{m}^{q_{m}} \leq\left(\sum_{m=1}^{n} q_{m} \alpha_{m}^{p}\right)^{1 / p}
$$

where $p \geq 0, \alpha_{m} \geq 0$, and $q_{m}>0$ for $m \in \mathbb{N}$ with $\sum_{m=1}^{n} q_{m}=1$.

Let $c_{m}>0, \alpha_{m}=c_{m} a_{m}$, and $q_{m}=\lambda_{m} / \Lambda_{m}$, then we obtain

$$
\left(c_{1} a_{1}\right)^{\lambda_{1} / \Lambda_{n}}\left(c_{2} a_{2}\right)^{\lambda_{2} / \Lambda_{n}} \cdots\left(c_{n} a_{n}\right)^{\lambda_{n} / \Lambda_{n}} \leq\left(\frac{1}{\Lambda_{n}} \sum_{m=1}^{n} \lambda_{m}\left(c_{m} a_{m}\right)^{p}\right)^{1 / p} .
$$

Further, we have

$$
\begin{aligned}
& \sum_{n=1}^{\infty} \lambda_{n+1}\left(a_{1}^{\lambda_{1}} a_{2}^{\lambda_{2}} \cdots a_{n}^{\lambda_{n}}\right)^{1 / \Lambda_{n}} \\
& \quad=\sum_{n=1}^{\infty} \lambda_{n+1} \frac{\left(c_{1} a_{1}\right)^{\lambda_{1} / \Lambda_{n}}\left(c_{2} a_{2}\right)^{\lambda_{2} / \Lambda_{n}} \cdots\left(c_{n} a_{n}\right)^{\lambda_{n} / \Lambda_{n}}}{\left(c_{1}^{\lambda_{1}} c_{2}^{\lambda_{2}} \cdots c_{n}^{\lambda_{n}}\right)^{1 / \Lambda_{n}}} \\
& \quad \leq \sum_{n=1}^{\infty} \frac{\lambda_{n+1}}{\left(c_{1}^{\lambda_{1}} c_{2}^{\lambda_{2}} \cdots c_{n}^{\lambda_{n}}\right)^{1 / \Lambda_{n}}}\left(\frac{1}{\Lambda_{n}} \sum_{m=1}^{n} \lambda_{m}\left(c_{m} a_{m}\right)^{p}\right)^{1 / p} .
\end{aligned}
$$

By the following inequality (see $[3,10])$

$$
\left(\sum_{m=1}^{n} z_{m}\right)^{t} \leq t \sum_{m=1}^{n} z_{m}\left(\sum_{k=1}^{m} z_{k}\right)^{t-1}
$$

where $t \geq 1$ is constant and $z_{m} \geq 0$ for $m \in \mathbb{N}$, it is easy to see that

$$
\begin{aligned}
\left(\frac{1}{\Lambda_{n}} \sum_{m=1}^{n} \lambda_{m}\left(c_{m} a_{m}\right)^{p}\right)^{1 / p} & \leq \frac{1}{\Lambda_{n}}\left(\sum_{m=1}^{n} \lambda_{m}\left(c_{m} a_{m}\right)^{p}\right)^{1 / p} \\
& \leq \frac{1}{p \Lambda_{n}} \sum_{m=1}^{n} \lambda_{m}\left(c_{m} a_{m}\right)^{p}\left(\sum_{k=1}^{m} \lambda_{k}\left(c_{k} a_{k}\right)^{p}\right)^{(1-p) / p},
\end{aligned}
$$

where $\Lambda_{n} \geq 1$ and $0<p \leq 1$. Thus, we obtain from (3.3) and (3.5) that

$$
\begin{aligned}
& \sum_{n=1}^{\infty} \lambda_{n+1}\left(a_{1}^{\lambda_{1}} a_{2}^{\lambda_{2}} \cdots a_{n}^{\lambda_{n}}\right)^{1 / \Lambda_{n}} \\
& \quad \leq \frac{1}{p} \sum_{n=1}^{\infty} \frac{\lambda_{n+1}}{\Lambda_{n}\left(c_{1}^{\lambda_{1}} c_{2}^{\lambda_{2}} \cdots c_{n}^{\lambda_{n}}\right)^{1 / \Lambda_{n}}} \sum_{m=1}^{n} \lambda_{m}\left(c_{m} a_{m}\right)^{p}\left(\sum_{k=1}^{m} \lambda_{k}\left(c_{k} a_{k}\right)^{p}\right)^{(1-p) / p} \\
& \quad=\frac{1}{p} \sum_{m=1}^{\infty} \lambda_{m}\left(c_{m} a_{m}\right)^{p} \sum_{n=m}^{\infty}\left(\frac{\lambda_{n+1}}{\Lambda_{n}\left(c_{1}^{\lambda_{1}} c_{2}^{\lambda_{2}} \cdots c_{n}^{\lambda_{n}}\right)^{1 / \Lambda_{n}}}\right)\left(\sum_{k=1}^{m} \lambda_{k}\left(c_{k} a_{k}\right)^{p}\right)^{(1-p) / p} .
\end{aligned}
$$


Choosing $c_{1}^{\lambda_{1}} c_{2}^{\lambda_{2}} \cdots c_{n}^{\lambda_{n}}=\left(\Lambda_{n+1}\right)^{\Lambda_{n}}$ for $n \in \mathbb{N}$ and setting $\Lambda_{0}=0$, we get from $0<\lambda_{n+1} \leq$ $\lambda_{n}$ that

$$
c_{n}=\left[\frac{\left(\Lambda_{n+1}\right)^{\Lambda_{n}}}{\left(\Lambda_{n}\right)^{\Lambda_{n-1}}}\right]^{1 / \lambda_{n}}=\left(1+\frac{\lambda_{n+1}}{\Lambda_{n}}\right)^{\Lambda_{n} / \lambda_{n}} \Lambda_{n} \leq\left(1+\frac{\lambda_{n}}{\Lambda_{n}}\right)^{\Lambda_{n} / \lambda_{n}} \Lambda_{n}
$$

This implies that

$$
\begin{aligned}
\sum_{n=1}^{\infty} \lambda_{n+1}\left(a_{1}^{\lambda_{1}} a_{2}^{\lambda_{2}} \cdots a_{n}^{\lambda_{n}}\right)^{1 / \Lambda_{n}} & \\
& \leq \frac{1}{p} \sum_{m=1}^{\infty} \lambda_{m}\left(c_{m} a_{m}\right)^{p} \sum_{n=m}^{\infty} \frac{\lambda_{n+1}}{\Lambda_{n} \Lambda_{n+1}}\left(\sum_{k=1}^{m} \lambda_{k}\left(c_{k} a_{k}\right)^{p}\right)^{(1-p) / p} \\
& =\frac{1}{p} \sum_{m=1}^{\infty} \lambda_{m}\left(c_{m} a_{m}\right)^{p} \sum_{n=m}^{\infty}\left(\frac{1}{\Lambda_{n}}-\frac{1}{\Lambda_{n+1}}\right)\left(\sum_{k=1}^{m} \lambda_{k}\left(c_{k} a_{k}\right)^{p}\right)^{(1-p) / p} \\
& =\frac{1}{p} \sum_{m=1}^{\infty} \lambda_{m}\left(c_{m} a_{m}\right)^{p} \frac{1}{\Lambda_{m}}\left(\sum_{k=1}^{m} \lambda_{k}\left(c_{k} a_{k}\right)^{p}\right)^{(1-p) / p} \\
& \leq \frac{1}{p} \sum_{m=1}^{\infty}\left(1+\frac{1}{\Lambda_{m} / \lambda_{m}}\right)^{p \Lambda_{m} / \lambda_{m}} \lambda_{m} a_{m}^{p} \Lambda_{m}^{p-1}\left(\sum_{k=1}^{m} \lambda_{k}\left(c_{k} a_{k}\right)^{p}\right)^{(1-p) / p} .
\end{aligned}
$$

Hence, we obtain from the above inequality and Lemma 2.1 that

$$
\begin{aligned}
& \sum_{n=1}^{\infty} \lambda_{n+1}\left(a_{1}^{\lambda_{1}} a_{2}^{\lambda_{2}} \cdots a_{n}^{\lambda_{n}}\right)^{1 / \Lambda_{n}} \\
& \quad<\frac{e^{p}}{p} \sum_{n=1}^{\infty}\left(1-\frac{1-2 / e}{\Lambda_{n} / \lambda_{n}}\right)^{p} \lambda_{n} a_{n}^{p} \Lambda_{n}^{p-1}\left(\sum_{k=1}^{n} \lambda_{k}\left(c_{k} a_{k}\right)^{p}\right)^{(1-p) / p} .
\end{aligned}
$$

The last inequality holds strictly since the right-hand inequality of (2.3) is valid if and only if $n=1$. The proof is complete.

Acknowledgments. The authors are indebted to the anonymous referees for their much detailed comments and suggestions to improve this note. The first and third authors were supported in part by the National Natural Science Foundation of China Grant 10001016, Science Foundation for the Prominent Youth of Henan Province Grant 0112000200, Science Foundation of Henan Innovation Talents at Universities, and the Doctor Fund of Henan Polytechnic University, China. The second author was supported in part by the Research Grants Council of the Hong Kong SAR (project no. HKU7040/03P), China. 


\section{References}

[1] P. S. Bullen, Handbook of Means and Their Inequalities, Mathematics and Its Applications, vol. 560, Kluwer Academic Publishers, Dordrecht, 2003.

[2] A. Čižmešija, J. Pečarić, and L.-E. Persson, On strengthened weighted Carleman's inequality, Bull. Austral. Math. Soc. 68 (2003), no. 3, 481-490.

[3] G. S. Davies and G. M. Petersen, On an inequality of Hardy's. II, Quart. J. Math. Oxford Ser. (2) 15 (1964), 35-40.

[4] S. S. Dragomir and Y.-H. Kim, The strengthened Hardy inequalities and its new generalizations, RGMIA Res. Rep. Coll. 4 (2001), no. 4, Article 2, http://rgmia.vu.edu.au/ v4n4.html.

[5] G. H. Hardy, Notes on some points in the integral calculus, Messenger of Math. 54 (1925), 150156.

[6] G. H. Hardy, J. E. Littlewood, and G. Pólya, Inequalities, 2nd ed., Cambridge University Press, Cambridge, 1952.

[7] M. Johansson, L.-E. Persson, and A. Wedestig, Carleman's inequality-history, proofs and some new generalizations, JIPAM. J. Inequal. Pure Appl. Math. 4 (2003), no. 3, Article 53, 1-19, http://jipam.vu.edu.au/article.php?sid=291.

[8] S. Kaijser, L.-E. Persson, and A. Öberg, On Carleman and Knopp's inequalities, J. Approx. Theory 117 (2002), no. 1, 140-151.

[9] A. Kufner and L.-E. Persson, Weighted Inequalities of Hardy Type, World Scientific Publishing, New Jersey, 2003.

[10] J. Németh, Generalizations of the Hardy-Littlewood inequality, Acta Sci. Math. (Szeged) 32 (1971), 295-299.

[11] Z. Xie and Y. Zhong, A best approximation for constant e and an improvement to Hardy's inequality, J. Math. Anal. Appl. 252 (2000), no. 2, 994-998.

[12] P. Yan and G. Sun, A strengthened Carleman's inequality, J. Math. Anal. Appl. 240 (1999), no. 1, 290-293.

[13] B. Yang, On Hardy's inequality, J. Math. Anal. Appl. 234 (1999), no. 2, 717-722.

[14] B. Yang and L. Debnath, Some inequalities involving the constant e, and an application to Carleman's inequality, J. Math. Anal. Appl. 223 (1998), no. 1, 347-353.

[15] X. Yang, On Carleman's inequality, J. Math. Anal. Appl. 253 (2001), no. 2, 691-694.

Chao-Ping Chen: Department of Applied Mathematics and Informatics, Research Institute of Applied Mathematics, Henan Polytechnic University, Jiaozuo, Henan 454000, China

E-mail addresses: chenchaoping@hpu.edu.cn; chenchaoping@sohu.com

Wing-Sum Cheung: Department of Mathematics, The University of Hong Kong, Pokfulam Road, Hong Kong

E-mail address: wscheung@hkucc.hku.hk

Feng Qi: Department of Applied Mathematics and Informatics, Research Institute of Applied Mathematics, Henan Polytechnic University, Jiaozuo, Henan 454000, China

E-mail addresses: qifeng@hpu.edu.cn; fengqi618@member.ams.org 


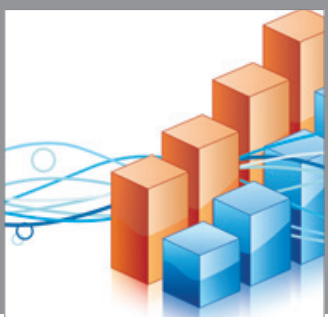

Advances in

Operations Research

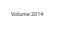

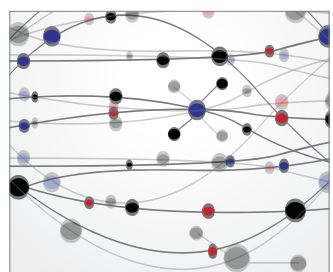

\section{The Scientific} World Journal
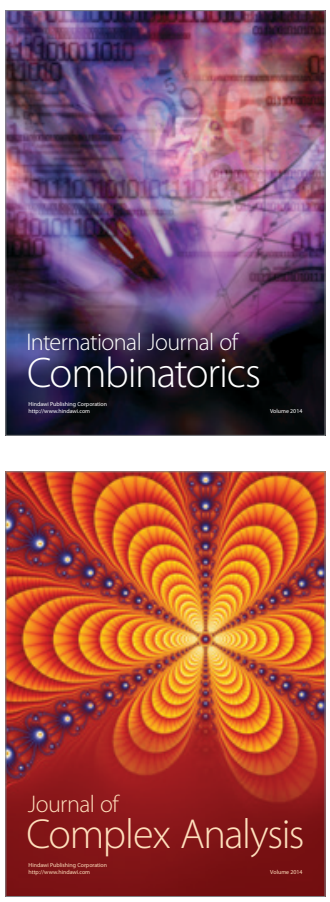

International Journal of

Mathematics and

Mathematical

Sciences
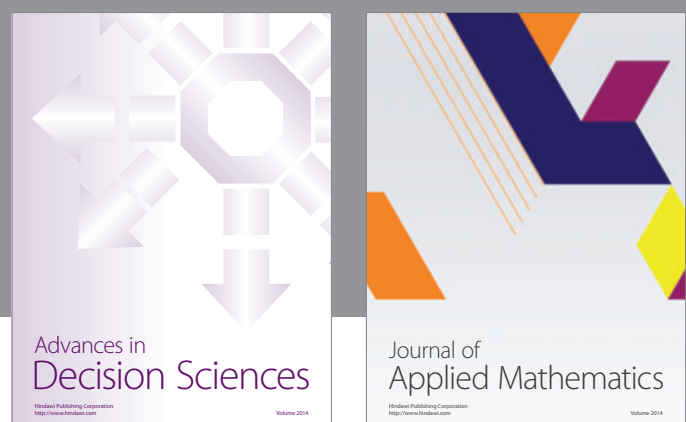

Journal of

Applied Mathematics
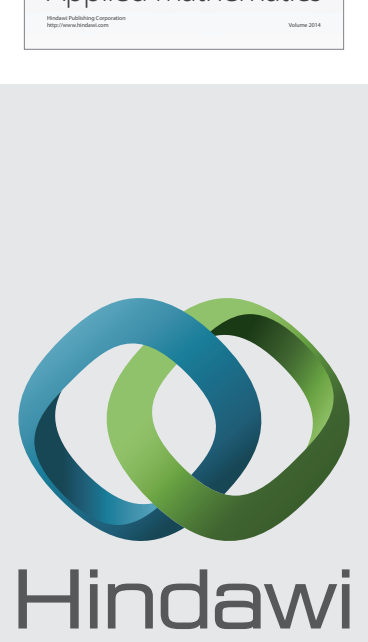

Submit your manuscripts at http://www.hindawi.com
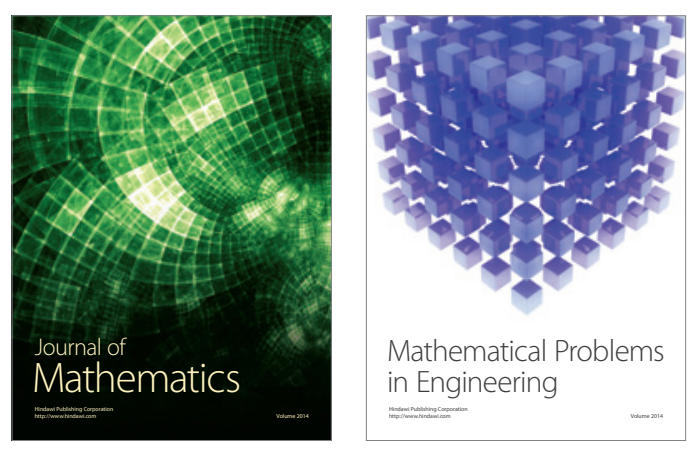

Mathematical Problems in Engineering
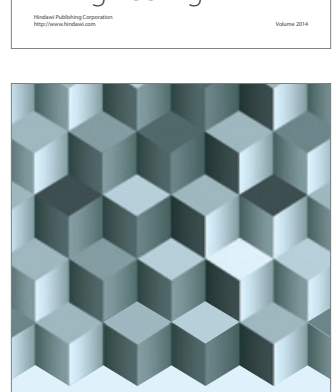

Journal of

Function Spaces
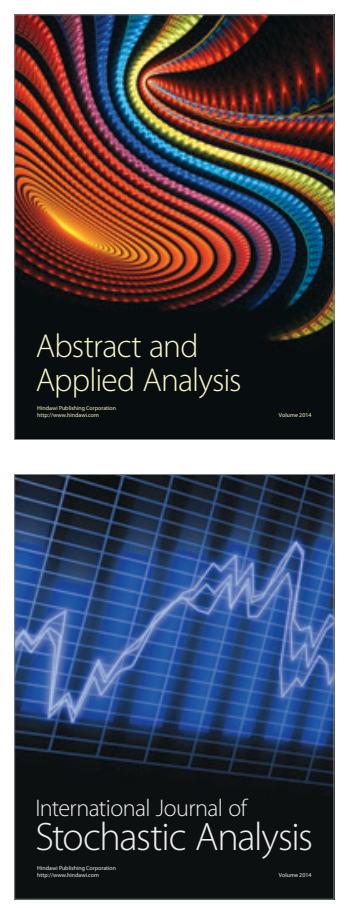

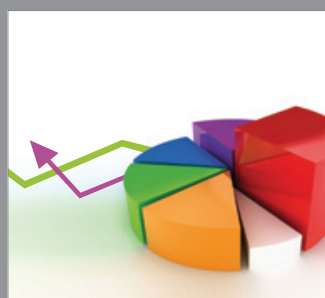

ournal of

Probability and Statistics

Promensencen
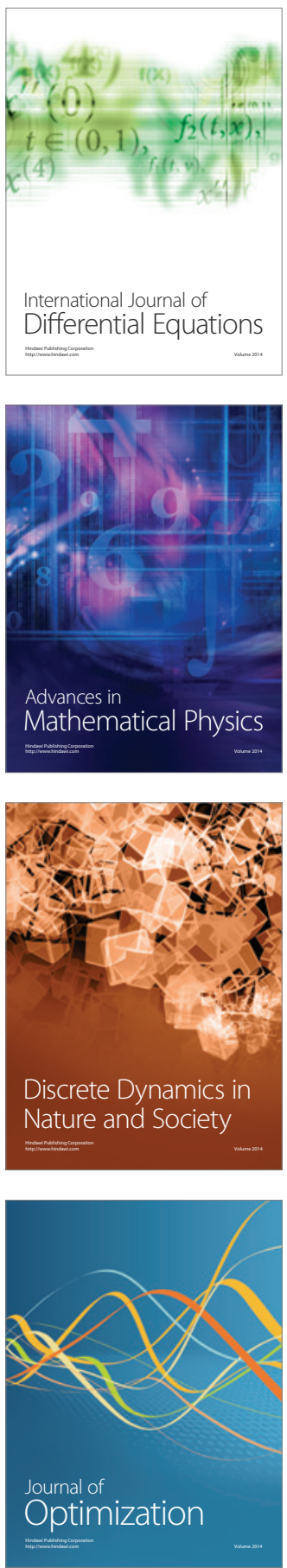